\title{
Gröbner bases for the polynomial ring with infinite variables and their applications
}

\author{
Kei-ichiro Iima and Yuji Yoshino
}

\begin{abstract}
We develop the theory of Gröbner bases for ideals in a polynomial ring with countably infinite variables over a field. As an application we reconstruct some of the one-one correspondences among various sets of partitions by using division algorithm.
\end{abstract}

\section{Introduction}

The purpose of this paper is to develop the theory of Gröbner bases for ideals in a polynomial ring $k\left[x_{1}, x_{2}, \ldots\right]$ with countably infinite variables over a field $k$. In such a case, ideals are not necessarily finitely generated, and hence the Gröbner bases for ideals might be consisting of infinite polynomials. However we shall claim that there is still an algorithm to get the Gröbner base for a given ideal.

This idea of Gröbner bases for infinitely generated ideals is strongly motivated by the following observation. Recall that a sequence $\lambda=$ $\left(\lambda_{1}, \lambda_{2}, \ldots, \lambda_{r}\right)$ of positive integers is called a partition of a non-negative integer $n$ if the equality $\lambda_{1}+\lambda_{2}+\cdots+\lambda_{r}=n$ holds and $\lambda_{1} \geq \lambda_{2} \geq \cdots \geq \lambda_{r} \geq 1$. In such a case we denote it by $\lambda \vdash n$. We are concerned with the following sets of partitions:

$$
\begin{aligned}
& A(n)=\left\{\begin{array}{llll}
\lambda \vdash n & \lambda_{i} \equiv \pm 1 \quad(\bmod 6)
\end{array}\right\}, \\
& B(n)=\left\{\begin{array}{lll}
\lambda \vdash n & \lambda_{i} \equiv \pm 1 \quad(\bmod 3), \quad \lambda_{1}>\lambda_{2}>\cdots>\lambda_{r}
\end{array}\right\}, \\
& C(n)=\left\{\begin{array}{ll|l}
\lambda \vdash n & \text { each } \lambda_{i} \text { is odd, and }
\end{array}\right. \\
& \text { any number appears in } \left.\lambda_{i}{ }^{\prime} \text { 's at most two times }\right\} \text {. }
\end{aligned}
$$

It is known by the famous Schur's equalities (see [1]) that all these sets $A(n)$, $B(n)$ and $C(n)$ have the same cardinality for all $n \in \mathbb{N}$. It is also known that 
the one-to-one correspondences among these three sets are realized in some combinatorial way using 2-adic or 3-adic expansions of integers. However such one-to-one correspondences can be reconstructed through the division algorithm by using the theory of Gröbner bases. For this, we need to extend the theory of Gröbner bases to a polynomial ring with infinitely many variables.

In Section 1, we shall give necessary definitions of initial ideals, Gröbner bases, S-polynomials and regular sequences in the polynomial ring $k\left[x_{1}, x_{2}, \ldots\right]$. And we develop the theory of Gröbner bases for ideals in such a polynomial ring by presenting a sequence of propositions, most of which goes in parallel with the ordinary case for ideals in polynomial rings with finitely many variables. But the difference is that ideals are not necessarily finitely generated and we need to argue about infinite set of polynomials as Gröbner bases and regular sequences. One of the essentially new results in this paper is Theorem 1.12 where we give an algorithm to get the reduced Gröbner bases. The other one is Theorem 1.22 in which we show that any permutation of a homogeneous regular sequence of infinite length is again a regular sequence.

In Section 2, we apply the theory developed in Section 1 to the sets of partitions. The main result is Theorem 2.1, where we give one-to-one correspondences between various sets of partitions by using division algorithm in the theory of Gröbner bases. As one of the applications we shall give the bijective mapping among the above mentioned sets $A(n), B(n)$ and $C(n)$.

\subsection{Gröbner bases for ideals}

Throughout this paper, let $k$ be any field and let $S=k\left[x_{1}, x_{2}, \ldots\right]$ be a polynomial ring with countably infinite variables. We denote by $\mathbb{Z}_{\geq 0}^{(\infty)}$ the set of all sequences $a=\left(a_{1}, a_{2}, \ldots\right)$ of integers where $a_{i}=0$ for all $i$ but finite number of integers. Also we denote by $\operatorname{Mon}(S)$ the set of all monomials in $S$. Since any monomial is described uniquely as $x^{a}=\prod_{i} x_{i}^{a_{i}}$ for some $a=\left(a_{1}, a_{2}, \ldots\right) \in \mathbb{Z}_{\geq 0}^{(\infty)}$, we can identify these sets, i.e. $\operatorname{Mon}(S) \cong \mathbb{Z}_{\geq 0}^{(\infty)}$. If we attach degree on $S$ by $\operatorname{deg} x_{i}=d_{i}$, then a monomial $x^{a}$ has degree $\operatorname{deg} x^{a}=\sum_{i=1}^{\infty} a_{i} d_{i}$. In the rest of the paper, we assume that the degrees $d_{i}$ 's are chosen in such a way that there are only a finite number of monomials of degree $d$ for each $d \in \mathbb{N}$. For example, the simplest way of attaching degree is that $\operatorname{deg} x_{i}=i$ for all $i \in \mathbb{N}$.

Definition 1.1. A total order $>$ on $\operatorname{Mon}(S)$ is called a monomial order if $(\operatorname{Mon}(S),>)$ is a well-ordered set, and it is compatible with the 
multiplication of monomials, i.e. $x^{a}>x^{b}$ implies $x^{c} x^{a}>x^{c} x^{b}$ for all $x^{a}, x^{b}, x^{c} \in \operatorname{Mon}(S)$. (See [4, Chapter 15].)

Note that the order $x_{1}>x_{2}>x_{3}>\cdots$ is not acceptable for monomial order, since it violates the well-ordering condition. On the other hand, if we are given any monomial order $>$, then, renumbering the variables, we may assume that $x_{1}<x_{2}<x_{3}<\cdots$.

The following are examples of monomial orders on $\operatorname{Mon}(S)$. (See [4, Chapter 15, pp. 329-330].)

Example 1.2. Let $a=\left(a_{1}, a_{2}, \ldots\right)$ and $b=\left(b_{1}, b_{2}, \ldots\right)$ be elements in $\mathbb{Z}_{\geq 0}^{(\infty)}$.

(1) The pure lexicographic order $>_{p l}$ is defined in such a way that $x^{a}>_{p l} x^{b}$ if and only if $a_{i}>b_{i}$ for the last index $i$ with $a_{i} \neq b_{i}$.

(2) The homogeneous (resp. anti-) lexicographic order $>_{h l}$ (resp. $>_{h a l}$ ) is defined in such a way that $x^{a}>_{h l} x^{b}$ (resp. $x^{a}>_{h a l} x^{b}$ ) if and only if either $\operatorname{deg} x^{a}>\operatorname{deg} x^{b}$ or $\operatorname{deg} x^{a}=\operatorname{deg} x^{b}$ and $a_{i}>b_{i}$ for the last (resp. first) index $i$ with $a_{i} \neq b_{i}$.

(3) The homogeneous (resp. anti-) reverse lexicographic order $>_{h r l}$ (resp. $>_{\text {harl }}$ ) is defined as follows: $x^{a}>_{h r l} x^{b}$ (resp. $\left.x^{a}>_{h a r l} x^{b}\right)$ if and only if either $\operatorname{deg} x^{a}>\operatorname{deg} x^{b}$ or $\operatorname{deg} x^{a}=\operatorname{deg} x^{b}$ and $a_{i}<b_{i}$ for the first (resp. last) index $i$ with $a_{i} \neq b_{i}$.

As in the orders in (2) to (3), if it satisfies that $\operatorname{deg} x^{a}>\operatorname{deg} x^{b}$ implies $x^{a}>x^{b}$, then we say that the order $>$ is homogeneous. The monomial orders in Example 1.2 are all distinct as shown in the following example in which $\operatorname{deg} x_{i}=i$ for $i \in \mathbb{N}$ :

$$
\begin{array}{ccccccccc}
x_{4} & >_{h l} & x_{1} x_{3} & >_{h l} & x_{2}^{2} & >_{h l} & x_{1}^{2} x_{2} & >_{h l} & x_{1}^{4}, \\
x_{1}^{4} & >_{h a l} & x_{1}^{2} x_{2} & >_{h a l} & x_{1} x_{3} & >_{h a l} & x_{2}^{2} & >_{h a l} & x_{4}, \\
x_{4} & >_{h r l} & x_{2}^{2} & >_{h r l} & x_{1} x_{3} & >_{h r l} & x_{1}^{2} x_{2} & >_{h r l} & x_{1}^{4}, \\
x_{1}^{4} & >_{h a r l} & x_{1}^{2} x_{2} & >_{h a r l} & x_{2}^{2} & >_{h a r l} & x_{1} x_{3} & >_{h a r l} & x_{4} .
\end{array}
$$

Now suppose that a monomial order $>$ on $\operatorname{Mon}(S)$ is given and we fix it in the rest of this section. Then, any non-zero polynomial $f \in S$ is expressed as

$$
f=c_{1} x^{a(1)}+c_{2} x^{a(2)}+\cdots+c_{r} x^{a(r)},
$$

where $c_{i} \neq 0 \in k$ and $x^{a(1)}>x^{a(2)}>\ldots>x^{a(r)}$. In such a case, the leading term, the leading monomial and the leading coefficient of $f$ are given respectively as $\ell t(f)=c_{1} x^{a(1)}, \ell m(f)=x^{a(1)}$ and $\ell c(f)=c_{1}$. For a non-zero 
ideal $I \subset S$, the initial ideal $\operatorname{in}(I)$ of $I$ is defined to be the ideal generated by all the leading terms $\ell t(f)$ of non-zero polynomials $f \in I$. (See 4, Chapter 15, p. 329].)

For a positive integer $n$, we set $S^{\langle n\rangle}=k\left[x_{1}, x_{2}, \ldots, x_{n}\right]$ which is a polynomial subring of $S$. Note that there is a filtration $S^{\langle 1\rangle} \subset \cdots \subset S^{\langle n\rangle} \subset$ $S^{\langle n+1\rangle} \subset \cdots \subset S$ and $S=\cup_{n=1}^{\infty} S^{\langle n\rangle}$. Since $\operatorname{Mon}\left(S^{\langle n\rangle}\right) \subset \operatorname{Mon}(S)$, we always employ the restricted monomial order from $\operatorname{Mon}(S)$ as a monomial order on $\operatorname{Mon}\left(S^{\langle n\rangle}\right)$. Therefore if $f \in S$ then the leading monomial of $f$ in $S^{\langle n\rangle}$ is independent of any such $n$ with $f \in S^{\langle n\rangle}$.

One easily observes the following remark. (See [4, Chapter 15, Proposition 15.4].)

Remark 1.3. (1) Assume that the monomial order is a pure lexicographic order. If $\ell m(f) \in S^{\langle n\rangle}$ for $f \in S$ and $n \in \mathbb{N}$, then $f \in S^{\langle n\rangle}$.

(2) Assume that the monomial order is a homogeneous lexicographic order. If $\ell m(f) \in S^{\langle n\rangle}$ for a homogeneous polynomial $f \in S$ and $n \in \mathbb{N}$, then $f \in S^{\langle n\rangle}$.

(3) Assume that the monomial order is a homogeneous reverse lexicographic order. If $\ell m(f) \in\left(x_{1}, x_{2}, \ldots, x_{n}\right) S$ for a homogeneous polynomial $f \in S$ and $n \in \mathbb{N}$, then $f \in\left(x_{1}, x_{2}, \ldots, x_{n}\right) S$.

The Gröbner base for an ideal of $S$ is defined similarly to the ordinary case. (See [4, Chapter 15].)

Definition 1.4. A subset $\mathcal{G}$ of an ideal $I$ of $S$ is called a Gröbner base for $I$ if $\{\operatorname{lm}(g) \mid g \in \mathcal{G}\}$ generates the initial ideal $i n(I)$.

It is easily observed that a Gröbner base for $I$ is actually a generating set of $I$. Note that an ideal $I$ does not necessarily admit a finite Gröbner base, since $S$ is not a Noetherian ring. However the generating set of $i n(I)$ is a subset of $\operatorname{Mon}(S)$ which must be a countable set, hence one can always take a countable set of polynomials as a Gröbner base for $I$.

Any argument concerning Gröbner bases for an ideal of $S$ can be reduced to the ordinary case for the polynomial rings with finite variables by the following lemma, in which, for a subset $\mathcal{G}$ of $S$, we denote by $\operatorname{in}(\mathcal{G})$ the set of all the leading monomials $\operatorname{lm}(g)$ for $g \in \mathcal{G}$.

Lemma 1.5. The following conditions are equivalent for a subset $\mathcal{G}$ of an ideal $I$ of $S$.

(1) $\mathcal{G}$ is a Gröbner base for I. 
(2) in $(\mathcal{G}) \cap S^{\langle n\rangle}$ generates the initial ideal in $\left(I \cap S^{\langle n\rangle}\right)$ for all integers $n$.

(3) in $(\mathcal{G}) \cap S^{\langle n\rangle}$ generates the initial ideal in $\left(I \cap S^{\langle n\rangle}\right)$ for infinitely many integers $n$.

Proof. $(1) \Rightarrow(2)$ : Suppose $\mathcal{G}$ is a Gröbner base for $I$ and let $f \in I \cap S^{\langle n\rangle}$. Then there is $g \in \mathcal{G}$ such that $\ell m(g)$ divides $\ell m(f)$. Since $\ell m(f) \in S^{\langle n\rangle}$, we have $\operatorname{lm}(g) \in \operatorname{in}(\mathcal{G}) \cap S^{\langle n\rangle}$. Thus in $(\mathcal{G}) \cap S^{\langle n\rangle}$ generates in $\left(I \cap S^{\langle n\rangle}\right)$.

$(2) \Rightarrow(3)$ : Trivial.

$(3) \Rightarrow(1)$ : Let $f \in I$ be any element. Take an integer $n$ so that $f \in S^{\langle n\rangle}$. Then, by the condition (3), there is an integer $m \geq n$ such that $i n\left(I \cap S^{\langle m\rangle}\right)$ is generated by $\operatorname{in}(\mathcal{G}) \cap S^{\langle m\rangle}$. Since $f \in I \cap S^{\langle m\rangle}$, the leading monomial $\ell m(f)$ is a multiple of $\operatorname{lm}(g)$ for some $g \in \mathcal{G}$. Therefore $\mathcal{G}$ is a Gröbner base for $I$.

Corollary 1.6. Let $\mathcal{G}$ be a subset of an ideal I of $S$. Assume that $\mathcal{G} \cap S^{\langle n\rangle}$ is a Gröbner base for an ideal $I \cap S^{\langle n\rangle}$ for infinitely many integers $n$. Then $\mathcal{G}$ is a Gröbner base for $I$.

Proof. It follows from the definition that $\operatorname{in}\left(\mathcal{G} \cap S^{\langle n\rangle}\right) \subseteq i n(\mathcal{G}) \cap S^{\langle n\rangle}$. Since $\operatorname{in}\left(\mathcal{G} \cap S^{\langle n\rangle}\right)$ generates the initial ideal $\operatorname{in}\left(I \cap S^{\langle n\rangle}\right)$ for such infinitely many integers $n$ in the assumption, $\mathcal{G}$ is a Gröbner base for $I$ by Lemma 1.5 .

Note that the inclusion $i n\left(\mathcal{G} \cap S^{\langle n\rangle}\right) \subseteq i n(\mathcal{G}) \cap S^{\langle n\rangle}$ is strict in general.

Corollary 1.7. Assume that the monomial order is a pure lexicographic order. If $\mathcal{G}$ is a Gröbner base for an ideal I of $S$, then $\mathcal{G} \cap S^{\langle n\rangle}$ is a Gröbner base for $I \cap S^{\langle n\rangle}$ for all $n \in \mathbb{N}$.

Proof. It follows from Remark 1.3 (1) that the equality in $\left(\mathcal{G} \cap S^{\langle n\rangle}\right)=$ in $(\mathcal{G}) \cap$ $S^{\langle n\rangle}$ holds in this case and it is a generating set of $i n\left(I \cap S^{\langle n\rangle}\right)$ for each $n$.

Now we can construct a Gröbner base for any ideal of $S$.

Proposition 1.8. Let $I$ be an ideal of $S$ and let $C$ be an arbitrary infinite subset of $\mathbb{N}$. For each $n \in C$, take a Gröbner base $\mathcal{G}_{n}$ for an ideal $I \cap S^{\langle n\rangle}$ inside $S^{\langle n\rangle}$. Then, the set $\bigcup_{n \in C} \mathcal{G}_{n}$ is a Gröbner base for $I$.

Proof. Set $\mathcal{G}=\bigcup_{n \in C} \mathcal{G}_{n}$, and we see that $\mathcal{G} \cap S^{\langle n\rangle}$ contains $\mathcal{G}_{n}$ for each $n \in C$, hence $\mathcal{G} \cap S^{\langle n\rangle}$, as well as $\mathcal{G}_{n}$, is a Gröbner base for $I \cap S^{\langle n\rangle}$ for such $n$. Hence Corollary 1.6 implies that $\mathcal{G}$ is a Gröbner base for $I$.

Compare the following division algorithm with that in [4, Chapter 15, Proposition-Definition 15.6]. 
Proposition 1.9 (Division algorithm). Let $\mathcal{G}$ be a subset of $S$. Then any non-zero polynomial $f \in S$ has an expression

$$
f=f_{1} g_{1}+f_{2} g_{2}+\cdots+f_{s} g_{s}+f^{\prime},
$$

with $g_{i} \in \mathcal{G}$ and $f_{i}, f^{\prime} \in S$ such that the following conditions hold:

(1) If we write $f^{\prime}=\sum_{i=1}^{t} c_{i} x^{a(i)}$ with $c_{i} \neq 0 \in k$, then $x^{a(i)} \notin$ in $(\mathcal{G} S)$ for each $i=1,2, \ldots, t$.

(2) If $f_{i} g_{i} \neq 0$, then $\operatorname{lm}\left(f_{i} g_{i}\right) \leq \ell m(f)$.

Any such $f^{\prime}$ is called a remainder of $f$ with respect to $\mathcal{G}$. Note that a remainder is in general not necessarily unique. But if $\mathcal{G}$ is a Gröbner base for $I=\mathcal{G} S$, then a remainder of $f$ with respect to $\mathcal{G}$ is uniquely determined.

Proof. The existence of such an expression is proved by induction on $\ell m(f)$, which goes in a similar way to the proof in [4, Proposition 15.8]. In fact, if $\ell m(f) \in i n(\mathcal{G S})$, then one can find $g \in \mathcal{G}$ whose leading monomial divides $\ell m(f)$, i.e. $\ell t(f)=c \mu \cdot \ell t(g)$ for some monomial $\mu$ and $c \in k$. In this case, since $\ell m(f-c \mu g)<\ell m(f)$, the proof is done by the induction hypothesis. If $\ell m(f) \notin i n(\mathcal{G} S)$, then arguing about $f-\ell t(f)$ we will have a desired expression again by the induction hypothesis.

The last half of the proposition is obvious from the definition of Gröbner bases.

Let us assume that $S$ is a graded ring with homogeneous monomial order and $\mathcal{G}$ is a set of homogeneous polynomials. Then we remark that if $f \in$ $S$ is a homogenous polynomial, then all polynomials in the expression in Proposition 1.9 can be taken to be homogeneous, hence the remainder of $f$ with respect to $\mathcal{G}$ is also homogenous.

By virtue of Proposition 1.9, the membership problem has a solution as in the ordinary cases.

Corollary 1.10. Let $\mathcal{G}$ be a Gröbner base for an ideal I. Then an element $f \in S$ belongs to $I$ if and only if 0 is the remainder of $f$ with respect to $\mathcal{G}$.

Recall that a Gröbner base $\mathcal{G}$ for a non-zero ideal $I$ of $S$ is called a reduced Gröbner base if every $g \in \mathcal{G}$ is a monic polynomial, i.e. $\ell c(g)=1$, and $\ell m(g)$ does not divide any term of $h$ for any $g \neq h \in \mathcal{G}$. Note that any ideal of $S^{\langle n\rangle}$ has a unique reduced Gröbner base. (See [6, Chapter 1].)

Proposition 1.11. For an arbitrary non-zero ideal I of $S$, there uniquely exists a reduced Gröbner base for $I$. 
Proof. Let $\left\{\mu_{\lambda} \mid \lambda \in \Lambda\right\}$ be a minimal generating set of the monomial ideal in $(I)$, i.e. it generates $i n(I)$ and any monomial dividing properly $\mu_{\lambda}$ does not belong to $i n(I)$. It is easy to see that such a minimal generating set uniquely exists for $\mathrm{in}(I)$. Take $g_{\lambda} \in I$ such that $\operatorname{lm}\left(g_{\lambda}\right)=\mu_{\lambda}$, and set $\mathcal{G}=\left\{g_{\lambda} \mid \lambda \in \Lambda\right\}$. Then it is clear that $\mathcal{G}$ is a Gröbner base for $I$. Replacing $g_{\lambda}$ with its remainder with respect $\mathcal{G} \backslash\left\{g_{\lambda}\right\}$, we can see that $\mathcal{G}$ is a reduced Gröbner base for $I$.

To prove the uniqueness, let $\mathcal{G}$ and $\mathcal{G}^{\prime}$ be reduced Gröbner bases for $I$. Assume that $\mathcal{G} \not \subset \mathcal{G}^{\prime}$. Then take $g \in \mathcal{G} \backslash \mathcal{G}^{\prime}$ so that $\ell m(g)$ is minimum among those polynomials in $\mathcal{G} \backslash \mathcal{G}^{\prime}$. Since $\mathcal{G}^{\prime}$ is a Gröbner base for $I$, there is $g^{\prime} \in \mathcal{G}^{\prime}$ such that $\ell m\left(g^{\prime}\right)$ divides $\ell m(g)$. Then it forces $\ell m(g)=\ell m\left(g^{\prime}\right)$, since $g$ is an element of a reduced Gröbner base. Note that every term of the polynomial $g-g^{\prime}$ is not belonging to $i n(\mathcal{G} S)$, since it is smaller than $\ell m(g)$ and since any monomial $\mu \in \mathcal{G}$ with $\mu<\ell m(g)$ belongs to $\mathcal{G}^{\prime}$. As a result, we see that $g-g^{\prime}$ is a remainder of $g-g^{\prime}$ itself with respect to $\mathcal{G}$. As we remarked above as the membership problem, this forces $g=g^{\prime}$ and hence $g \in \mathcal{G}^{\prime}$. This contradiction shows that $\mathcal{G} \subseteq \mathcal{G}^{\prime}$. And by the symmetry of arguments we conclude that $\mathcal{G}=\mathcal{G}^{\prime}$.

Theorem 1.12. Let I be a non-zero ideal I of $S$. Take a reduced Gröbner base $\mathcal{G}_{n}$ for $I \cap S^{\langle n\rangle}$ inside the polynomial ring $S^{\langle n\rangle}$ for each $n$, and consider the following set of polynomials in $S$;

$$
\overline{\mathcal{G}}=\bigcup_{m=1}^{\infty}\left(\bigcap_{n=m}^{\infty} \mathcal{G}_{n}\right) .
$$

Then $\overline{\mathcal{G}}$ is a reduced Gröbner base for $I$.

Proof. Let $\mathcal{G}$ be the unique reduced Gröbner base for $I$, whose existence we have shown in Proposition 1.11. First we prove that $\mathcal{G} \subseteq \overline{\mathcal{G}}$. To show this, let $g \in \mathcal{G}$ and take an integer $n$ so that $g \in S^{\langle n\rangle}$. Note that $\operatorname{lm}(g)$ is not divisible by any other monomial belonging to the minimal generating set of the monomial ideal $i n(I)$, and also note that any terms of $g$ other than $\ell t(g)$ are not divisible by any monomial in the minimal generating set of $i n(I)$. This implies that $g$ is a member of the reduced Gröbner base for $I \cap S^{\langle n\rangle}$, hence $g \in \mathcal{G}_{n}$ for such $n$. Therefore $g \in \overline{\mathcal{G}}$, and we have shown $\mathcal{G} \subseteq \overline{\mathcal{G}}$. Since $\overline{\mathcal{G}}$ contains a Gröbner base for $I$ and since $\overline{\mathcal{G}} \subseteq I, \overline{\mathcal{G}}$ is a Gröbner base for $I$ as well.

To show that $\overline{\mathcal{G}}$ is a reduced Gröbner base for $I$, let $g, h$ be distinct elements of $\overline{\mathcal{G}}$. Take an integer $m$ so that $g, h \in \bigcap_{n \geq m} \mathcal{G}_{n}$, in particular $g, h \in \mathcal{G}_{m}$. Since $\mathcal{G}_{m}$ is a reduced Gröbner base, we see that $\ell m(h)$ does not divide any term of $g$. Hence the Gröbner base $\overline{\mathcal{G}}$ is a reduced one. 
Recall that the $S$-polynomial of elements $f, g \in S$ are defined to be

$$
S(f, g)=\frac{L C M\{\ell m(f), \ell m(g)\}}{\ell t(f)} f-\frac{L C M\{\ell m(f), \ell m(g)\}}{\ell t(g)} g .
$$

(See [4, Chapter 15.4].) Note here that $S$ is a unique factorization domain and that the least common multiple $L C M$ is defined well. Now the Buchberger's criterion for Gröbner bases is proved in a similar way to ordinary cases for polynomial rings with finite variables [4, Theorem 15.8].

Proposition 1.13 (Buchberger's criterion). Let $\mathcal{G}$ be a generating subset of an ideal $I \subset S$. Then $\mathcal{G}$ is a Gröbner base for $I$ if and only if 0 is a remainder of $S\left(g_{\lambda}, g_{\mu}\right)$ with respect to $\mathcal{G}$ for all pairs $\left(g_{\lambda}, g_{\mu}\right)$ of elements in $\mathcal{G}$.

Proof. The "only if" part is obvious. To prove the "if" part, let $f \in I$ and we show that $\ell m(f)$ is a multiple of $\ell m(g)$ for an element $g \in \mathcal{G}$. Since $\mathcal{G}$ generates the ideal $I$, an equality $f=\sum_{i=1}^{r} h_{i} g_{i}$ holds for some $g_{i} \in \mathcal{G}$ and $h_{i} \in S(1 \leq i \leq r)$. Let $\mu$ be the monomial which is maximum among $\ell m\left(h_{i} g_{i}\right)(1 \leq i \leq r)$. If $\ell m(f)=\mu$, then there is nothing to prove, because $\ell m(f)=\ell m\left(h_{i} g_{i}\right)=\ell m\left(h_{i}\right) \ell m\left(g_{i}\right)$ for some $i$. If $\ell m(f)<\mu$, then applying the following claims to $f_{i}=\ell m\left(h_{i}\right) g_{i}$ and $\mu_{i}=\ell m\left(h_{i}\right)$ for those indices $i$ with $\ell m\left(h_{i} g_{i}\right)=\mu$, we shall have an alternative equality $f=\sum_{i=1}^{r^{\prime}} h_{i}^{\prime} g_{i}^{\prime}$ such that the maximum monomial $\mu^{\prime}$ is smaller than $\mu$, and the proof will be through.

Claim 1: Assume that $f_{1}, f_{2}, \ldots, f_{s} \in S$ are polynomials in $S$ having the same leading monomial $\mu$. If $\operatorname{lm}\left(\sum_{j=1}^{s} c_{j} f_{j}\right)<\mu$ holds for some $c_{j} \in k$, then $\sum_{j=1}^{s} c_{j} f_{j}$ is described as a linear combination of the S-polynomials $S\left(f_{j}, f_{\ell}\right) \quad(1 \leq j<\ell \leq s)$.

Claim 2: Let $f_{i_{1}}, f_{i_{2}}$ be non-zero elements of $S$ and let $\mu_{i_{1}}, \mu_{i_{2}}$ be monomials. If $\operatorname{lm}\left(\mu_{i_{1}} f_{i_{1}}\right)=\ell m\left(\mu_{i_{2}} f_{i_{2}}\right)$, then $S\left(\mu_{i_{1}} f_{i_{1}}, \mu_{i_{2}} f_{i_{2}}\right)=\mu_{i_{1}} f_{i_{1}}-\mu_{i_{2}} f_{i_{2}}$ is a multiple of $S\left(f_{i_{1}}, f_{i_{2}}\right)$.

Proofs of the claims are similar to the ordinary cases and we leave them to the reader.

In the following proposition, we assume that $S$ is a graded ring and that the monomial order is homogeneous.

Proposition 1.14. Let I be a homogeneous ideal of $S$, and let $\mathcal{G}$ be a Gröbner base for I consisting of homogeneous polynomials. Define $\varphi: S \rightarrow S$ by mapping $f \in S$ to the remainder of $f$ with respect to $\mathcal{G}$. Then $\varphi$ induces a mapping $\bar{\varphi}: S / I \rightarrow S /$ in $(I)$ which is an isomorphism as graded k-vector spaces. 
Proof. Since $\mathcal{G}$ is a Gröbner base, $\varphi(f)$ is uniquely determined for $f \in S$ by Proposition 1.9. If $\varphi(f) \in i n(I)$, then $\varphi(f)=0$ and it follows from Corollary 1.10 that $f \in I$. Hence $\bar{\varphi}$ is an injection. For any monomial $\mu \notin i n(I)$, it is clear that $\mu$ itself is a remainder of $\mu$ with respect to $\mathcal{G}$. Therefore $\bar{\varphi}$ is surjective.

Using the mapping $\bar{\varphi}$, one can construct a one-to-one correspondence between the $k$-bases of $S / I$ and $S / i n(I)$. This idea is a key for the argument in the next section.

Let $R$ be a residue ring of the graded polynomial ring $S$ by a homogeneous ideal $I$, i.e. $R=S / I$. Then $R$ is also a graded ring and we denote by $R_{n}$ the part of degree $n$ of $R$. Recall that the Hilbert series of $R$ is defined to be

$$
H_{R}(T)=\sum_{i=0}^{\infty}\left(\operatorname{dim}_{k} R_{n}\right) T^{n}
$$

which is an element of $\mathbb{Z}[[T]]$. Note that

$$
H_{S}(T)=\prod_{i=1}^{\infty} \frac{1}{1-T^{d_{i}}}
$$

where $d_{i}=\operatorname{deg}\left(x_{i}\right)$. Remark that by the definition of graded structure of $S$, each $S_{n}$, hence $R_{n}$, is of finite dimension over $k$. In particular, there are only a finite number of variables $x_{i}$ with $\operatorname{deg}\left(x_{i}\right) \leq n$ for each integer $n$. Hence $H_{R}(T)$ and $H_{S}(T)$ are well-defined elements of $\mathbb{Z}[[T]]$.

Proposition 1.14 implies the following equality for Hilbert series.

Corollary 1.15. Under the same assumption as the proposition, the equality $H_{S / I}(T)=H_{S / \text { in }(I)}(T)$ holds.

Now we are discussing about the regularity condition for a sequence of elements in the ring.

Definition 1.16. Let $R$ be an arbitrary ring in this definition. Let $\Omega$ be a well-ordered set and suppose we are given a set of elements $\mathcal{T}=\left\{f_{\alpha} \in\right.$ $R \mid \alpha \in \Omega\}$ indexed by $\Omega$.

(1) We call $\mathcal{T}$ a regular sequence on $R$ if $R /\left(f_{\alpha} \mid \alpha \in \Omega\right) R \neq 0$ and $f_{\alpha}$ is a non-zero divisor on $R /\left(f_{\beta} \mid \beta<\alpha\right) R$ for all $\alpha \in \Omega$. If $R$ is a graded ring, then a regular sequence consisting of homogeneous elements in $R$ is called a homogeneous regular sequence. 
(2) We say that the sequence $\mathcal{T}$ satisfies the FR-condition (the finite regularity condition), if any finite subsequences $\left\{f_{\alpha_{1}}, f_{\alpha_{2}}, \ldots, f_{\alpha_{r}}\right\}$ with $\alpha_{1}<\alpha_{2}<\ldots<\alpha_{r}$ in $\Omega$ is a regular sequence in this order.

We shall prove that these two regularity conditions above are equivalent for homogeneous sequences. One implication holds for any sequence and it is easily proved as in the following lemma.

Lemma 1.17. If an ordered set $\left\{f_{\alpha} \in R \mid \alpha \in \Omega\right\}$ satisfies the FR-condition, then it is a regular sequence.

Proof. If $R=\left(f_{\alpha} \mid \alpha \in \Omega\right) R$, then we have the equality $1=\sum_{i=1}^{r} f_{\alpha_{i}} h_{i}$ for some $f_{\alpha_{1}}, \ldots, f_{\alpha_{r}}$ and $h_{i} \in R$. Thus $R=\left(f_{\alpha_{1}}, \ldots, f_{\alpha_{r}}\right) R$ and this contradicts to the FR-condition. Therefore we have $R /\left(f_{\alpha} \mid \alpha \in \Omega\right) R \neq 0$.

Suppose that $h f_{\alpha} \in\left(f_{\beta} \mid \beta<\alpha\right) R$ for $h \in R$. Then we have the expression $h f_{\alpha}=\sum_{i=1}^{r} f_{\beta_{i}} h_{i}$ for some $\beta_{1}<\cdots<\beta_{r}<\alpha$ and $h_{i} \in R$. Since $\left\{f_{\beta_{1}}, \ldots, f_{\beta_{r}}, f_{\alpha}\right\}$ is a regular sequence in this order, we get $h \in\left(f_{\beta_{1}}, \ldots, f_{\beta_{r}}\right) R \subset\left(f_{\beta} \mid \beta<\alpha\right) R$.

To prove the other implication we need several lemmas.

Lemma 1.18. Let $\left\{f_{\alpha} \in R \mid \alpha \in \Omega\right\}$ be a regular sequence indexed by a well-ordered set $\Omega$, and set $I=\left(f_{\alpha} \mid \alpha \in \Omega\right) R$. Let $\mathbb{Y}=\left\{Y_{\alpha} \mid \alpha \in \Omega\right\}$ be a set of indeterminates over $R$ corresponding to $\Omega$. For a homogeneous polynomial $F \in R[\mathbb{Y}]_{d}$ of degree $d$ in $\mathbb{Y}$, we denote by $F(f)$ the elements of $R$ obtained by substituting $f_{\alpha}$ for $Y_{\alpha}$. Under this notation, if $F(f) \in I^{d+1}$, then $F \in I R[\mathbb{Y}]$.

Proof. Using a transfinite induction on $\alpha \in \Omega$ we shall prove a more strong statement:

Claim 1: Let $I_{\alpha}=\left(f_{\beta} \mid \beta \leq \alpha\right) R$ and $\mathbb{Y}_{\alpha}=\left\{Y_{\beta} \mid \beta \leq \alpha\right\}$. For a homogeneous polynomial $F \in R\left[\mathbb{Y}_{\alpha}\right]_{d}$, if $F(f) \in I_{\alpha}^{d+1}$, then $F \in I_{\alpha} R\left[\mathbb{Y}_{\alpha}\right]$.

As the transfinite induction hypothesis, we assume that Claim 1 holds for any $\alpha^{\prime} \in \Omega$ with $\alpha^{\prime}<\alpha$. To prove Claim 1 we need the following auxiliary result.

Claim 2: Let $J_{\alpha}=\left(f_{\beta} \mid \beta<\alpha\right) R$ and $\mathbb{Y}_{\alpha}^{\prime}=\left\{Y_{\beta} \mid \beta<\alpha\right\}$. Then $f_{\alpha}$ is a non-zero divisor on $R / J_{\alpha}^{j}$ for all $j \geq 1$.

In fact, suppose that $h f_{\alpha} \in J_{\alpha}^{j}$ for some $j>1$. Arguing by the induction on $j$ we have $h \in J_{\alpha}^{j-1}$, hence $h=H(f)$ for some $H \in R\left[\mathbb{Y}_{\alpha}^{\prime}\right]_{j-1}$. Thus $f_{\alpha} H(f)=f_{\alpha} h \in J_{\alpha}^{(j-1)+1}$, and applying the transfinite induction hypothesis 
to $f_{\alpha} H \in R\left[\mathbb{Y}_{\alpha}^{\prime}\right]_{j-1}$, we have $f_{\alpha} H \in J_{\alpha} R\left[\mathbb{Y}_{\alpha}^{\prime}\right]$. Since $f_{\alpha}$ is a non-zero divisor on $R / J_{\alpha}$, we have $H \in J_{\alpha} R\left[\mathbb{Y}_{\alpha}^{\prime}\right]_{j-1}$, therefore $h=H(f) \in J_{\alpha}^{j}$.

Now we proceed to the proof of Claim 1. For this, let $F \in R\left[\mathbb{Y}_{\alpha}\right]_{d}$. We shall prove by the induction on $d$ that $F(f) \in I_{\alpha}^{d+1}$ implies $F \in I_{\alpha} R\left[\mathbb{Y}_{\alpha}\right]$. If $d=0$ then the claim is trivial, hence we assume that $d>0$.

First we show that we may assume that $F(f)=0$. Since $F(f) \in I_{\alpha}^{d+1}$, there exists $G \in R\left[\mathbb{Y}_{\alpha}\right]_{d+1}$ such that $F(f)=G(f)$. Writing $G=\sum_{i=1}^{n} Y_{\beta_{i}} G_{i}$ with $\beta_{i} \leq \alpha$ and $G_{i} \in R\left[\mathbb{Y}_{\alpha}\right]_{d}$, we set $G^{\prime}=\sum_{i=1}^{n} f_{\beta_{i}} G_{i}$. Then we have $F-G^{\prime} \in R\left[\mathbb{Y}_{\alpha}\right]_{d}$ and $\left(F-G^{\prime}\right)(f)=0$. Furthermore, it holds that $F-G^{\prime} \in$ $I_{\alpha} R\left[\mathbb{Y}_{\alpha}\right]$ if and only if $F \in I_{\alpha} R\left[\mathbb{Y}_{\alpha}\right]$.

Henceforth we assume $F(f)=0$. Then we may write $F=G+Y_{\alpha} H$ with $G \in R\left[\mathbb{Y}_{\alpha}^{\prime}\right]_{d}$ and $H \in R\left[\mathbb{Y}_{\alpha}\right]_{d-1}$. Since $f_{\alpha} H(f)=-G(f) \in J_{\alpha}^{d}$, the Claim 2 above implies that $H(f) \in J_{\alpha}^{d} \subset I_{\alpha}^{(d-1)+1}$. Thus, by the induction on $d$, we get $H \in I_{\alpha} R\left[\mathbb{Y}_{\alpha}\right]$. On the other hand, since $H(f) \in J_{\alpha}^{d}$, there is $H^{\prime} \in R\left[\mathbb{Y}_{\alpha}^{\prime}\right]_{d}$ such that $H(f)=H^{\prime}(f)$ holds. As $\left(G+f_{\alpha} H^{\prime}\right)(f)=F(f)=0$, it follows by transfinite induction on $\alpha$ that $G+f_{\alpha} H^{\prime} \in J_{\alpha} R\left[\mathbb{Y}_{\alpha}^{\prime}\right] \subset I_{\alpha} R\left[\mathbb{Y}_{\alpha}\right]$. Since $f_{\alpha} H^{\prime} \in I_{\alpha} R\left[\mathbb{Y}_{\alpha}\right]$, we get $G \in I_{\alpha} R\left[\mathbb{Y}_{\alpha}\right]$. Therefore $F \in I_{\alpha} R\left[\mathbb{Y}_{\alpha}\right]$. This completes the proof.

Corollary 1.19. Let $\left\{f_{\alpha} \in R \mid \alpha \in \Omega\right\}$ be a regular sequence indexed by a well-ordered set $\Omega$, and set $I=\left(f_{\alpha} \mid \alpha \in \Omega\right) R$. And let $\mathbb{Y}=\left\{Y_{\alpha} \mid \alpha \in \Omega\right\}$ be a set of indeterminates over $R$ corresponding to $\Omega$ as in the lemma. Then the map $\varphi:(R / I)[\mathbb{Y}] \rightarrow \operatorname{gr}_{I}(R)=\bigoplus_{n=0}^{\infty} I^{n} / I^{n+1}$ induced by the substitution $Y_{\alpha} \mapsto \bar{f}_{\alpha} \in I / I^{2}$ is an isomorphism as algebras over $R / I$. In particular, $I^{n} / I^{n+1}$ is an $(R / I)$-free module for all $n \in \mathbb{N}$.

Proof. From the definition, $\varphi$ is a well-defined algebra map over $R / I$ that is surjective. It follows from Lemma 1.18 that $\varphi$ is injective as well.

Lemma 1.20. Let $R=\bigoplus_{n=0}^{\infty} R_{n}$ be a non-negatively graded ring, and let $\left\{f_{\alpha} \mid \alpha \in \Omega\right\}$ be a sequence of homogeneous elements of positive degree in $R$ indexed by a well-ordered set $\Omega$. If $\left\{f_{\alpha} \mid \alpha \in \Omega\right\}$ satisfies the FR-condition, then so does any permutation. More precisely, if $\Omega^{\prime}$ is another well-ordered set such that there is a bijective mapping $\sigma: \Omega^{\prime} \rightarrow \Omega$, then the sequence $\left\{f_{\sigma\left(\alpha^{\prime}\right)} \mid \alpha^{\prime} \in \Omega^{\prime}\right\}$ satisfies the FR-condition whenever $\left\{f_{\alpha} \mid \alpha \in \Omega\right\}$ does.

Proof. By the definition of the FR-condition it is enough to show that any permutation of a finite homogeneous regular sequence $f_{1}, f_{2}, \ldots, f_{r}$ is again a regular sequence. For this we have only to show that if $f, g$ is a homogeneous regular sequence, then so is $g, f$. Suppose that $f, g$ is a homogeneous regular sequence on $R$. Assume $h g=0$ for $h \in R$ and we want to show that $h=0$. We may assume that $h$ is homogeneous. Since $h g \in(f) R$, we see $h \in(f) R$. 
Writing $h=h_{1} f$ for some $h_{1} \in R$, we have $h_{1} g=0$ since $f$ is a non-zero divisor on $R$. Hence $h_{1} \in(f) R$, and $h \in\left(f^{2}\right) R$. Subsequently we have $h \in\left(f^{n}\right) R$ for any $n \geq 1$. Take $n$ so that $n \operatorname{deg} f>\operatorname{deg} h$ and we conclude that $h=0$.

Next assume $h^{\prime} f \in(g) R$ for $h^{\prime} \in R$. Writing $h^{\prime} f=g h_{1}^{\prime}$, we have $h_{1}^{\prime}=f h_{2}^{\prime}$ for some $h_{2} \in R$, since $g h_{1}^{\prime} \in(f) R$ and $g$ is a non-zero divisor on $R /(f) R$. Since $f$ is a non-zero divisor on $R$, we have $h^{\prime}=g h_{2}^{\prime} \in(g) R$ as desired.

Proposition 1.21. Let $R=\bigoplus_{n=0}^{\infty} R_{n}$ be a non-negatively graded ring, and let $\left\{f_{\alpha} \mid \alpha \in \Omega\right\}$ be a sequence of homogeneous elements of positive degree in $R$ indexed by a well-ordered set $\Omega$. If $\left\{f_{\alpha} \mid \alpha \in \Omega\right\}$ is a regular sequence on $R$, then it satisfies the FR-condition.

Proof. Suppose there is a finite sequence $\left\{f_{\alpha_{1}}, f_{\alpha_{2}}, \ldots, f_{\alpha_{r}}\right\}$ with $\alpha_{1}<\alpha_{2}<$ $\ldots<\alpha_{r}$ such that it is not a regular sequence. Take such a $\left\{f_{\alpha_{1}}, f_{\alpha_{2}}, \ldots, f_{\alpha_{r}}\right\}$ with $\alpha_{r}$ being minimum in $\Omega$. Note by this choice of $\alpha_{r}$ that $\left\{f_{\beta} \mid \beta<\alpha_{r}\right\}$ satisfies the FR-condition. After changing the order of first $(r-1)$ elements in the sequence we may assume the following:

(i) $\alpha_{1}, \ldots, \alpha_{r-1}$ are the first $(r-1)$ elements in $\Omega$,

(ii) $\left\{f_{\beta} \mid \beta<\alpha_{r}\right\}$ satisfies the FR-condition,

(iii) $\left\{f_{\beta} \mid \beta<\alpha_{r}\right\} \sqcup\left\{f_{\alpha_{r}}\right\}$ is a regular sequence,

(iv) $\left\{f_{\alpha_{1}}, f_{\alpha_{2}}, \ldots, f_{\alpha_{r}}\right\}$ is not a regular sequence.

Considering the residue ring $\bar{R}=R /\left(f_{\alpha_{1}}, \ldots, f_{\alpha_{r-1}}\right) R$, we have:

(i)' $\left\{\bar{f}_{\beta} \in \bar{R} \mid \alpha_{r-1}<\beta<\alpha_{r}\right\}$ satisfies the FR-condition,

(ii)' $\left\{\bar{f}_{\beta} \in \bar{R} \mid \alpha_{r-1}<\beta<\alpha_{r}\right\} \sqcup\left\{\bar{f}_{\alpha_{r}}\right\}$ is a regular sequence on $\bar{R}$,

(iii)' $\bar{f}_{\alpha_{r}}$ is a zero divisor on $\bar{R}$.

We show a contradiction from this setting. Set $J=\left(\bar{f}_{\beta} \mid \alpha_{r-1}<\beta<\right.$ $\left.\alpha_{r}\right) \bar{R}$. By (iii)' there is a non-zero homogeneous element $\bar{g} \in \bar{R}$ such that $\bar{g} \bar{f}_{\alpha_{r}}=0$. Then we can find $n \in \mathbb{N}$ such that $\bar{g} \in J^{n} \backslash J^{n+1}$. Since $\bar{f}_{\alpha_{r}}$ is a non-zero divisor on $\bar{R} / J$ by (ii)', and since $\bar{f}_{\alpha_{r}} \bar{g} \equiv 0$ in $J^{n} / J^{n+1}$, we apply Corollary 1.19 using (i)' and we conclude that $\bar{g} \in J^{n+1}$. This is a contradiction.

As a consequence of 1.17, 1.20 and 1.21 we have the following result. 
Theorem 1.22. Let $R=\bigoplus_{n=0}^{\infty} R_{n}$ be a non-negatively graded ring, and let $\mathcal{T}=\left\{f_{\alpha} \mid \alpha \in \Omega\right\}$ be a sequence of homogeneous elements of positive degree in $R$ indexed by a well-ordered set $\Omega$. Then $\mathcal{T}$ is a regular sequence on $R$ if and only if $\mathcal{T}$ satisfies the FR-condition. In particular, any permutation of a homogeneous regular sequence is again a regular sequence.

Now we return to the case for the polynomial ring $S=k\left[x_{1}, x_{2}, \ldots\right]$.

Lemma 1.23. (1) Let $\mathcal{T}$ be a set of monomials in $S$. Then $\mathcal{T}$ is a homogeneous regular sequence on $S$ if and only if any two monomials in $\mathcal{T}$ are coprime, i.e. any distinct elements $\mu_{1}$ and $\mu_{2}$ in $\mathcal{T}$ have no common divisor except units.

(2) Let $\mathcal{T}$ be a homogeneous regular sequence on $S$ and let $n$ be an integer.

(a) Then $\mathcal{T} \cap S^{\langle n\rangle}$ is a finite set consisting of at most $n$ elements.

(b) The set $\{f \in \mathcal{T} \mid \operatorname{deg}(f) \leq n\}$ is a finite set.

(c) $\mathcal{T}$ is a countable set.

Proof. It is easy to prove (1) and we leave it the reader. To prove (2)(a), note that $\mathcal{T} \cap S^{\langle n\rangle}$ is a homogeneous regular sequence on $S^{\langle n\rangle}$, since $S^{\langle n\rangle} \subset S$ is a faithfully flat ring extension. The graded $k$-algebra $S^{\langle n\rangle}$ has depth $n$, hence any homogeneous regular sequence on $S^{\langle n\rangle}$ has at most length $n$. It forces $\left|\mathcal{T} \cap S^{\langle n\rangle}\right| \leqq n$. For (2)(b), recall that the grading for $S$ is given in such a way that there are only a finite number of monomials of degree $n$ for each integer $n$. Therefore, for any integer $n>0$, there is an integer $m$ such that the equality $S_{n}=\left(S^{\langle m\rangle}\right)_{n}$ holds. Therefore the set $\{f \in \mathcal{T} \mid \operatorname{deg}(f) \leq n\}$ is a subset of $\mathcal{T} \cap S^{\langle m\rangle}$ that is a finite set. Since the equality $\mathcal{T}=\bigcup_{n=0}^{\infty}\{f \in$ $\mathcal{T} \mid \operatorname{deg}(f) \leq n\}$ holds, $\mathcal{T}$ is a countable set.

Proposition 1.24. Let $\mathcal{T}$ be a homogeneous regular sequence on $S$, and let $I$ be the homogeneous ideal generated by $\mathcal{T}$. Then the Hilbert series of the graded ring $S / I$ is given by

$$
H_{S / I}(T)=H_{S}(T) \cdot \prod_{f \in \mathcal{T}}\left(1-T^{\operatorname{deg}(f)}\right) .
$$

Proof. For a graded $S$-module $M=\bigoplus_{i=0}^{\infty} M_{i}$, we denote by $M_{\leq n}$ the $k$ subspace of $M$ spanned by homogeneous elements of degree at most $n$; $M_{\leq n}=\bigoplus_{i \leq n} M_{i}$. Now let $\left\{f_{1}, \ldots, f_{r}\right\}$ be the set of all the elements of $\mathcal{T}$ of degree at most $n$ which is a finite set by Lemma 1.23 (2). Then it is easy to see that $I_{\leq n}=\left(\left(f_{1}, \ldots, f_{r}\right) S\right)_{\leq n}$. Thus $(S / I)_{\leq n}=\left(S /\left(f_{1}, \ldots, f_{r}\right) S\right)_{\leq n}$. From the definition of graded structure of $S$, given an integer $n$, we have an integer 
$m$ with the equality $S_{\leq n}=\left(S^{\langle m\rangle}\right)_{\leq n}$. We can take such an integer $m$ as $S^{\langle m\rangle}$ contains $f_{1}, \ldots, f_{r}$. Therefore we have $(S / I)_{\leq n}=\left(S^{\langle m\rangle} /\left(f_{1}, \ldots, f_{r}\right) S^{\langle m\rangle}\right)_{\leq n}$. This implies that the difference $H_{S / I}(T)-H_{S\langle m\rangle /\left(f_{1}, \ldots, f_{r}\right) S\langle m\rangle}(T)$ belongs to $T^{n} \mathbb{Z}[[T]]$. Note that the equality

$$
H_{S^{\langle m\rangle} /\left(f_{1}, \ldots, f_{r}\right) S^{\langle m\rangle}}(T)=H_{S^{\langle m\rangle}}(T) \cdot \prod_{i=1}^{r}\left(1-T^{\operatorname{deg}\left(f_{i}\right)}\right)
$$

is known to hold by [4, Exercise 19.14], since $\left\{f_{1}, \ldots, f_{r}\right\}$ is a regular sequence on $S^{\langle m\rangle}$. Thus we have

$$
H_{S / I}(T)-H_{S}(T) \cdot \prod_{f \in \mathcal{T}, \operatorname{deg}(f) \leq n}\left(1-T^{\operatorname{deg}(f)}\right) \in T^{n} \mathbb{Z}[[T]] .
$$

Since this holds for any integer $n$, the proof is completed.

The criterion of Bayer-Stillman [4, Proposition 15.15] is generalized in the following form.

Proposition 1.25. Let $\mathcal{T}=\left\{f_{\alpha} \mid \alpha \in \Omega\right\}$ be a set of elements in $S$. Assume that $\left\{\operatorname{lm}\left(f_{\alpha}\right) \mid \alpha \in \Omega\right\}$ is a regular sequence on $S$. Then $\mathcal{T}$ is a regular sequence on $S$ and it is a Gröbner base for the ideal $\mathcal{T} S$.

Proof. To prove that $\mathcal{T}$ is a Gröbner base, we have only to show that 0 is a remainder of $S(f, g)$ with respect to $\mathcal{T}$ for any $f, g \in \mathcal{T}$. See Proposition 1.13. More strongly we can show that 0 is a remainder of $S(f, g)$ with respect to $\{f, g\}$, whenever $\ell m(f), \ell m(g)$ is a regular sequence on $S$. In fact, $S(f, g)=$ $\ell t(g) f-\ell t(f) g=-(g-\ell t(g)) f+(f-\ell t(f)) g$ holds and it is easy to see that this description shows that the remainder is 0 .

Now to prove that $\mathcal{T}$ is a regular sequence on $S$, we assume that $h f_{\alpha} \in$ $\left(f_{\beta} \mid \beta<\alpha\right) S$ for $h \in S$ and $\alpha \in \Omega$. We shall show that $h \in\left(f_{\beta} \mid \beta<\alpha\right) S$ by the induction on $\ell m(h)$. Then we have $\ell m(h) \ell m\left(f_{\alpha}\right) \in i n\left(\left(f_{\beta} \mid \beta<\alpha\right) S\right)$. Since $\left\{\operatorname{lm}\left(f_{\beta}\right) \mid \beta<\alpha\right\}$ is a regular sequence on $S$, it follows from the first half of the proof that $\left\{f_{\beta} \mid \beta<\alpha\right\}$ is a Gröbner base for the ideal $\left(f_{\beta} \mid \beta<\alpha\right) S$. Thus there is a monomial $\operatorname{lm}\left(f_{\beta_{1}}\right)$ with $\beta_{1}<\alpha$ which divides $\ell m(h)$. Therefore $\ell m\left(h-c_{1} \mu_{1} f_{\beta_{1}}\right)<\ell m(h)$ holds for some $c_{1} \in k$ and a monomial $\mu_{1}$. Then it follows from the induction hypothesis that $h-c_{1} \mu_{1} f_{\beta_{1}} \in\left(f_{\beta} \mid \beta<\alpha\right) S$, hence $h \in\left(f_{\beta} \mid \beta<\alpha\right) S$.

\section{Applications}

Let $S=k\left[x_{1}, x_{2}, \ldots\right]$ be a polynomial ring with countably infinite variables as before. We regard $S$ as a graded $k$-algebra by defining $\operatorname{deg}\left(x_{i}\right)=i$ for 
each $i \in \mathbb{N}$, and denote by $S_{n}$ the part of degree $n$ of $S$ for $n \in \mathbb{N}$. Note that there is a bijective mapping between the set of partitions of $n$ and the set of monomials of degree $n$. The correspondence is given by mapping a partition $\lambda=\left(\lambda_{1}, \lambda_{2}, \ldots, \lambda_{r}\right) \vdash n$ to the monomial $x^{\lambda}=x_{\lambda_{r}} \cdots x_{\lambda_{2}} x_{\lambda_{1}}$ of degree $n$.

Let $W$ be any subset of $\mathbb{N}$ satisfying $p W \subset W$ for an integer $p \geq 2$, where $p W=\{p w \mid w \in W\}$. In this case, we consider a subring $R=k\left[X_{i} \mid i \in W\right]$ of $S$. We are interested in the following two subsets of partitions of $n$ :

$$
\begin{aligned}
& X(n)=\left\{\lambda \vdash n \quad \mid \quad \lambda_{i} \in W \backslash p W\right\}, \\
& Y(n)=\left\{\lambda \vdash n \quad \mid \quad \lambda_{i} \in W \text {, and any number appears among the } \lambda_{i}\right. \text { 's } \\
& \text { at most } p-1 \text { times } \text {. }
\end{aligned}
$$

Theorem 2.1. Under the circumstances above, consider the set of homogeneous polynomials $\mathcal{G}=\left\{x_{i}^{p}-x_{p i} \mid i \in W\right\}$ in $R$. We adopt the homogeneous anti-reverse lexicographic order (resp. the homogeneous lexicographic order) on the set of monomials in $R$. Then $\mathcal{G}$ is a reduced Gröbner base (resp. a Gröbner base) for the ideal $\mathcal{G} R$.

Furthermore, define a mapping $\varphi: X(n) \rightarrow Y(n)$ so that $x^{\varphi(\lambda)}$ is the remainder of $x^{\lambda}$ with respect to $\mathcal{G}$ in the homogeneous anti-reverse lexicographic order for any $\lambda \in X(n)$. Then $\varphi$ is a well-defined bijective mapping.

Proof. Note that $x_{i}^{p}>_{h a r l} x_{p i}\left(\operatorname{resp} . x_{p i}>_{h l} x_{i}^{p}\right)$, hence we have $\ell m\left(x_{i}^{p}-x_{p i}\right)=$ $x_{i}^{p}$ in homogeneous anti-reverse lexicographic order (resp. $\ell m\left(x_{i}^{p}-x_{p i}\right)=x_{p i}$ in homogeneous lexicographic order ) for all $i \in W$. Since it is clear that $\left\{x_{i}^{p} \mid i \in W\right\}$ (resp. $\left\{x_{p i} \mid i \in W\right\}$ ) is a homogeneous regular sequence on $R$, it follows from Proposition 1.25 that $\left\{x_{i}^{p}-x_{p i} \mid i \in W\right\}$ is a homogeneous regular sequence on $R$, which is a Gröbner base. Actually this is a reduced Gröbner base in the case of homogeneous anti-reverse lexicographic order.

To prove the second half of the theorem, let $\lambda \in X(n)$ be an arbitrary element. By definition $x^{\lambda}$ contains no variables $x_{p i}(i \in W)$. In order to get the remainder of $x^{\lambda}$ with respect to $\mathcal{G}$ in the homogeneous anti-reverse lexicographic order, we replace $x_{i}^{p}$ with $x_{p i}$ in the monomial, whenever $x^{\lambda}$ involves a $p$ th power $x_{i}^{p}$ of a variable. Continue this procedure until we get the monomial $x^{\rho}$ involving no $p$ th power of a variable. It is then clear that $\rho \in Y(n)$ and $x^{\rho}$ is the remainder of $x^{\lambda}$ with respect to $\mathcal{G}$ in the homogeneous anti-reverse lexicographic order. In such a way we have $\varphi(\lambda)=\rho$, hence the mapping $\varphi: X(n) \rightarrow Y(n)$ is well-defined.

In a similar manner to this, we can define $\psi: Y(n) \rightarrow X(n)$ by using the homogeneous lexicographic order and by replacing $x_{p i}$ with $x_{i}^{p}$ in the monomials, and it is obvious by the construction that $\varphi \cdot \psi=i d_{Y(n)}$ and $\psi \cdot \varphi=i d_{X(n)}$. 
Just considering the generating functions of $|X(n)|$ and $|Y(n)|$, we see that the following equality holds;

$$
\prod_{m \in W \backslash p W} \frac{1}{1-t^{m}}=\prod_{m \in W}\left(1+t^{m}+t^{2 m}+\cdots+t^{(p-1) m}\right) .
$$

Example 2.2. Recall that $A(n), B(n)$ and $C(n)$ are the sets of partitions given in Introduction.

(1) If $W=\{n \in \mathbb{N} \mid n \equiv \pm 1(\bmod 3)\}$ and $p=2$, then $X(n)=A(n)$ and $Y(n)=B(n)$.

(2) If $W=\{n \in \mathbb{N} \mid n \equiv 1(\bmod 2)\}$ and $p=3$, then $X(n)=A(n)$ and $Y(n)=C(n)$.

As a consequence of all the above, we obtain one-to-one correspondences among $A(n), B(n)$ and $C(n)$ by using the theory of Gröbner bases. Considering their generating functions we have the following equalities:

$$
\prod_{m \equiv \pm 1} \frac{1}{1-t^{m}}=\prod_{m \equiv \pm 1}\left(1+t^{m}\right),=\prod_{m \equiv 1}\left(1+t_{(\bmod 3)}(1) t^{2 m}\right)
$$

which are called the Schur's equalities. See [1, (1.2) and (1.3)].

We close the paper by raising a problem. For this let us consider the following sets of partitions.

$$
\begin{aligned}
& P(n)=\left\{\begin{array}{l|l}
\lambda \vdash n & \lambda_{i} \equiv \pm 1 \quad(\bmod 5)
\end{array}\right\}, \\
& Q(n)=\left\{\begin{array}{l|l}
\lambda \vdash n & \lambda_{i}-\lambda_{i+1} \geq 2
\end{array}\right\} .
\end{aligned}
$$

By Rogers-Ramanujan equality

$$
\prod_{m \equiv \pm 1} \frac{1}{1-t^{m}}=1+\sum_{m=1}^{\infty} \frac{t^{m^{2}}}{(1-t)\left(1-t^{2}\right) \cdots\left(1-t^{m}\right)}
$$

it is known that the sets $P(n)$ and $Q(n)$ have the same cardinality for each $n \in \mathbb{N}$. (See [2, (5.26)].)

If we find an ideal $I$ as in the following problem, then we will obtain a oneto-one correspondence between $P(n)$ and $Q(n)$ by using division algorithm.

Problem 2.3. Find an ideal $I$ of $S$ and a monomial order on $\operatorname{Mon}(S)$ satisfying $S / I \cong k\left[\left\{x_{i} \mid i \equiv \pm 1(\bmod 5)\right\}\right]$ and $\operatorname{in}(I)=\left(x_{i}^{2}, x_{i} x_{i+1} \mid i \in \mathbb{N}\right)$. 


\section{References}

[1] G. E. Andrews, Schur's theorem, partitions with odd parts and the Al-Salam-Carlitz polynomials. q-series from a contemporary perspective. (South Hadley, MA, 1998), 45-56, Contemp. Math., 254, Amer. Math. Soc., Providence, RI, 2000.

[2] D. M. Bressoud, Proofs and confirmations. The story of the alternating sign matrix conjecture. MAA Spectrum. Mathematical Association of America, Washington, DC; Cambridge University Press, Cambridge, 1999.

[3] W. Bruns and J. Herzog, Cohen-Macaulay rings. Cambridge Studies in Advanced Mathematics 39, Cambridge University Press, Cambridge, 1993.

[4] D. Eisenbud, Commutative algebra. With a view toward algebraic geometry. Graduate Texts in Mathematics 150, Springer-Verlag, New York, 1995.

[5] H. Matsumura, Commutative ring theory. Translated from the Japanese by M. Reid. Second edition. Cambridge Studies in Advanced Mathematics 8, Cambridge University Press, Cambridge, 1989.

[6] B. Sturmfels, Gröbner bases and convex polytopes. University Lecture Series, 8, American Mathematical Society, Providence, RI, 1996.

Kei-ichiro Iima; Graduate School of Natural Science and Technology,

Okayama University, Okayama 700-8530, Japan

(e-mail:iima@math.okayama-u.ac.jp)

Yuji Yoshino; Department of Math., Okayama University, Okayama 700-8530, Japan (e-mail:yoshino@math.okayama-u.ac.jp) 